\title{
NÍVEL DE ATIVIDADE FÍSICA DE ACORDO COM OS BAIRROS DA CIDADE DE SÃO CAETANO DO SUL, SP
}

\author{
LEVEL OF PHYSICAL ACTIVITY IN THE NEIGHBORHOODS \\ OF THE CITY OF SÃO CAETANO DO SUL, SÃO PAULO, BRAZIL
}

\section{Josivaldo De Souza Lima ${ }^{a^{*}}$, Victor Keihan Matsudo ${ }^{b^{* *}}$, Manoel Carlos Sampaio Ribeiro ${ }^{c^{* * *}}$}

\author{
ajosivaldoesporte@gmail.com, ${ }^{b}$ matsudo@celafiscs.org.br, cmcrmacal@gmail.com \\ *Centro de Estudos do Laboratório de Aptidão Física de São Caetano do Sul - São Caetano do Sul (SP), Brasil; \\ Faculdade de Ciências Médicas da Santa Casa de São Paulo - São Paulo (SP), Brasil \\ **Centro de Estudos do Laboratório de Aptidão Física de São Caetano do Sul - São Caetano do Sul (SP), Brasil \\ ***Faculdade de Ciências Médicas da Santa Casa de São Paulo - São Paulo (SP), Brasil
}

Data de recebimento do artigo: 12/03/2015

Data de aceite do artigo: 17/09/2015

\section{RESUMO}

O objetivo deste estudo foi verificar a prevalência do nível de atividade física nos 15 bairros da cidade de São Caetano do Sul na população acima de 18 anos de idade. A amostra foi composta por 669 indivíduos, sendo $51,9 \%$ do sexo feminino com média de idade de $48,1 \pm 17,9$, escolaridade de $9,0 \pm 4,9$ e renda de $\mathrm{R} \$$ $1.341,6 \pm 1.919$. Os dados são provenientes do inquérito populacional realizado anualmente pelo IMES da Universidade Municipal de São Caetano do Sul e no Instituto de Pesquisa (Inpes) do IMES. Para coleta de dados foi utilizado, nas entrevistas em domicílio, o International Physical Activity Questionnaire (IPAQ), versão curta, e para determinar o nível de atividade física, utilizou-se o consenso entre o Centro de Estudos do Laboratório de Aptidão Física de São Caetano do Sul (CELAFISCS) e o Centers for Disease Control and Prevention (CDC), que classifica a atividade física em quatro categorias: "muito ativo", "ativo", "irregularmente ativo" e "sedentário". $\mathrm{Na}$ análise estatística utilizamos o teste de Kolmogorov-Smirnov para verificar a normalidade dos dados, o teste ANOVA para verificar diferenças estatísticas e o teste de Bonferroni para localizar as diferenças entre a classificação do nível de atividade física de acordo com os bairros. O nível de confiança adotado foi $\mathrm{p}<0,05$. Com base nos dados analisados concluímos que não apresentaram diferenças estatisticamente significantes entre o nível de atividade física de acordo com os bairros estudados, entretanto, foi possível observar diferenças entre as classes socioeconômicas e entre a escolaridade e nível de atividade física.

Palavra-chave: Prevalência; atividade física; jovens; adultos e idosos.

\section{ABSTRACT}

The aim of this study was to determine the prevalence of physical activity in the 15 neighborhoods in the city of São Caetano do Sul (Brazil) in the population above 18 years of age. The sample consisted of 669 subjects, $51.9 \%$ female with a mean age of $48.1 \pm 17.9$, with educational background of $9.0 \pm 4.9$ and real income of BRL 1,341.6 $\pm 1,919$. The data are from a population survey conducted annually by the Municipal Institute for Higher Education (IMES) of the Municipal University of São Caetano do Sul and the IMES' Research Institute (INPES). In household interviews, the short version of the International Physical Activity Questionnaire (IPAQ) was used for data collection, and to determine the level of physical activity we used the consensus between the São Caetano do Sul's Physical Aptitude Laboratory Study Center (CELAFISCS) and the CDC, which classifies physical activity into four categories: "very active", "active", "irregularly active", and "sedentary". In the statistical analysis we used the Kolmogorov-Smirnov test to check the normality of the data. The ANOVA test was used to verify statistical differences and the Bonferroni test to locate the differences on the classification of the level of physical activity according to the neighborhoods. The confidence level was set at $\mathrm{p}<0.05$. Based on the analyzed data, we concluded that there were no statistically significant differences between the level of physical activity among the studied neighborhoods. However, it was possible to observe differences among socioeconomic classes and between educational background and level of activity.

Keywords: Prevalence; physical activity; youth; adults and elderly. 


\section{Introdução}

A prática regular da atividade física vem sendo estudada fortemente como um importante fator de proteção "modificável" contra doenças crônico-degenerativas que, ainda nos dias atuais, são uma das principais causas de morte em países desenvolvidos ${ }^{1}$.

Atribui-se a falta de atividade física a cerca de 5,3 milhōes de mortes anuais em todo o planeta. Segundo a Organização Mundial da Saúde (OMS), o sedentarismo está no ranking dos cincos fatores que mais matam na atualidade ${ }^{2}$. Considerado o mal do século, esse sedentarismo pode ser combatido nos níveis: intrapessoal, ambiente social e no ambiente físico ${ }^{3}$. Por isso, um programa de promoção à atividade física que não centre as ações no indivíduo tem mais chances de sucesso, visto que o indivíduo faz parte do meio onde convive ${ }^{4}$.

Na cidade de São Paulo na década de 1990, a primeira pesquisa realizada com grande abrangência sobre as questóes dos fatores de risco de doenças crônicas não transmissíveis já chamava atenção das autoridades públicas sobre a falta de atividade física dos paulistanos, que na época ambos os sexos já apresentavam alta prevalência de sedentarismo, no sexo masculino $57,3 \%$ e no feminino $80,2 \% 5$.

Quase 20 anos depois pouco mudou. Dados do Sistema de Vigilância de Fatores de Risco e Proteção para Doenças Crônicas por Inquérito Telefônico (Vigitel), do Ministério da Saúde, mostraram estabilidade nos níveis de atividade física no lazer de 2006 a 2009 e pouca evolução positiva nas demais ${ }^{6}$.

$\mathrm{Na}$ capital paulista, segundo dados do Vigitel apenas $28 \%$ da população realiza pelo menos 150 minutos de atividade física, de leve a moderada, ou pelo menos 75 minutos de atividade com intensidade vigorosa ${ }^{7}$.

No Brasil, dados populacionais de atividade física estáo surgindo timidamente. Uma pesquisa que analisou a atividade física no período de 2002 a 2007 na cidade de Pelotas, RS, mostrou uma tendência de diminuição na proporção de ativos ${ }^{8}$. Já na capital paulista outra análise do período de 2002 a 2008 mostrou diminuição no número de sedentários especialmente pelo aumento da caminhada? .

Com o objetivo de minimizar os efeitos do sedentarismo para a saúde, a OMS passou a recomendar atividades físicas regulares. A atual recomendação para adultos de 18 a 64 anos é de pelo menos 150 minutos de atividades aeróbicas moderadas ou 75 minutos de atividades aeróbicas intensas ${ }^{10,11}$. Essas atividades não necessariamente necessitam ser realizadas de uma única forma, também podem ser incluídas atividades, tais como locomoção, atividades domésticas, jogos, esportes ou exercícios planejados.
Entretanto, no Brasil dados de prevalência do nível de atividade física em cidades com alto Índice de Desenvolvimento Humano (IDH) são raros na literatura. Tendo isso em vista, o objetivo desta pesquisa foi analisar a prevalência de atividade física nos 15 bairros da cidade de São Caetano do Sul, considerada a cidade com melhor IDH do Brasil.

\section{Métodos}

Dados transversais dos inquéritos populacionais realizados anualmente na cidade de Sáo Caetano do Sul pelo Instituto de Pesquisa do Ensino Superior (IMPES). O sorteio sistemático dos domicílios foi realizado utilizando o cadastro de imóveis residenciais do município, com base no banco de dados do Imposto Territorial Urbano, a partir do número de inscrição imobiliária. No domićlio, os entrevistados tinham idade mínima de 18 anos. Os dados foram coletados mediante aplicação de questionário estruturado, a partir de entrevista pessoal, com duraçáo média de 60 minutos. Nesta pesquisa foram utilizados os dados disponíveis do último inquérito realizado em 2007, dos 15 bairros da cidade de Sáo Caetano do Sul. A amostra foi composta por 699 indivíduos. O nível de atividade física foi avaliado pelo International Physical Activity Questionnaire (IPAQ), versão curta, validado no Brasil ${ }^{12}$. Os participantes foram entrevistados e depois de responderem o questionário foram classificados em quatro categorias: "muito ativo", "ativo", "irregularmente ativo" e "sedentário".

\section{Análise estatística}

$\mathrm{Na}$ análise estatística utilizamos o teste de Kolmogorov-Smirnov para verificar a normalidade dos dados, o teste ANOVA para verificar diferenças estatísticas e o teste de Bonferroni para localizar as diferenças. O nível de confiança adotado foi $\mathrm{p}<0,05$.

\section{Resultados}

A amostra final contemplou 669 indivíduos do inquérito de 2007, os homens tinham uma média de idade de $47,3 \pm 18,4$ e as mulheres $49,1 \pm 16,2$, assim, não apresentaram diferenças significativas entre os sexos. Entretanto, encontramos diferenças estatísticas entre homens e mulheres no grupo ativo, indicando proporcionalmente ser maior a quantidade de mulheres ativas, representando 45,8\%, $<<0,05$. Quando subdividimos o 
grupo de acordo com anos de escolaridade encontramos uma proporção maior de pessoas com a escolaridade de 1 a 4 anos no grupo muito ativo, sendo 65,6\%, p>0,05. Tentamos ainda identificar se existiam diferenças de acordo com a classificação de atividade física entre os bairros, mas na nossa análise os bairros não apresentaram diferenças significativas. Embora o bairro Boa Vista tenha apresentado a menor proporção de indivíduos sedentários $(6,1 \%)$, esse valor não foi estatisticamente significante, nem mesmo quando comparado com o bairro proporcionalmente mais sedentário, São José, que apresentou 23,4\% de sedentários (Gráfico 1). Já o bairro
Cerâmica teve a menor proporçáo de ativos, apenas $22,1 \%$, enquanto o bairro Centro apresentou a maior proporçáo de ativos (45,7\%) (Gráfico 2). Na tabela 3 apresentamos a porcentagem e o intervalo de confiança do nível de atividade física da população de São Caetano do Sul de acordo com os bairros. Destacamos que os bairros que apresentaram maior número de sedentários foram São José com 23,4\% (IC 21,3-25,5) seguido de Santo Antônio com 21,7\% (IC 19,6-23,8). Já os bairros com maior proporção de ativos foram Centro com $47,8 \%$ (IC 45,7-49,8) seguido do bairro Nova Gerty com 47,7\% (IC 45,3-49,4).

Gráfico 1: Proporção de indivíduos classificados como sedentários de acordo com os bairros.

\section{Proporção de sedentários}

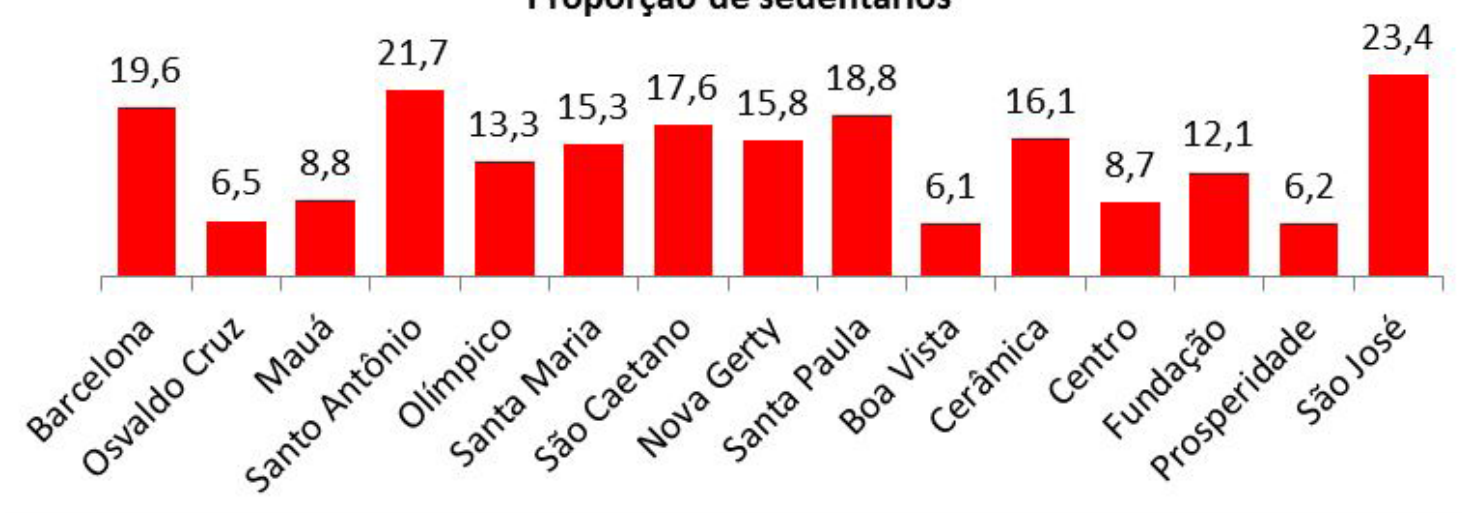

Gráfico 2: Proporção de indivíduos classificados como ativos de acordo com os bairros.

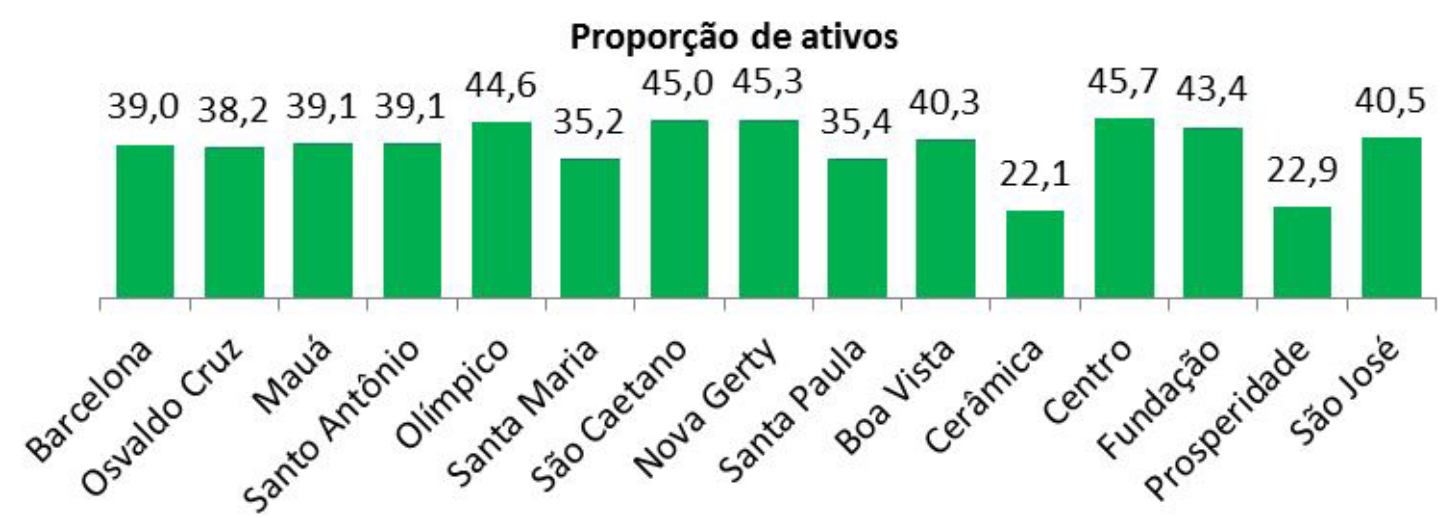

A Tabela 1 mostra a diferença do nível de atividade física entre os sexos. Encontramos diferenças apenas entre o grupo ativo, mostrando que no grupo de mulheres ativas a proporção foi de $45,8 \%$, sendo $11,6 \%$ a mais que os homens.
Já na Tabela 2 é apresentado o nível de atividade física de acordo com a escolaridade. A proporçáo de pessoas no grupo muito ativo e com escolaridade entre 1 a 4 anos foi $65,6 \%$ do total sendo diferente apenas do grupo com $>12$ anos de escolaridade, que apresentou apenas 6,6\%. 
Tabela 1: Distribuição da amostra de acordo com o sexo e nível de atividade física.

\begin{tabular}{cccccccccc} 
& \multicolumn{3}{c}{ Total } & \multicolumn{3}{c}{ Homem } & \multicolumn{2}{c}{ Mulher } \\
& $\%$ & \multicolumn{2}{c}{ IC 95\% } & $\%$ & IC 95\% & \% & IC 95\% \\
\hline Sedentário & 14,8 & 12,72 & 16,87 & 16,5 & 14,42 & 18,07 & 13,3 & 11,22 & 15,37 \\
\hline Irregularmente ativo & 22,4 & 20,32 & 24,47 & 23,0 & 20,92 & 25,07 & 21,9 & 19,82 & 23,97 \\
\hline Ativo & 40,2 & 38,12 & 42,27 & 34,2 & 32,12 & 36,27 & $45,8^{*}$ & 43,72 & 47,87 \\
\hline Muito ativo & 22,6 & 20,52 & 24,67 & 26,4 & 24,32 & 28,47 & 19,0 & 16,92 & 21,07 \\
\hline
\end{tabular}

${ }^{*} \mathrm{p}<0,05$ diferença entre homem e mulher no grupo ativo.

Tabela 2: Nível de atividade física de acordo com os anos de escolaridade.

\begin{tabular}{|c|c|c|c|c|c|c|c|c|c|c|c|c|c|c|c|}
\hline \multirow{3}{*}{$\begin{array}{c}\text { Anos de estudo } \\
\text { NAF } \\
\text { Sedentário }\end{array}$} & \multicolumn{3}{|c|}{0} & \multicolumn{3}{|c|}{$1-4$} & \multicolumn{3}{|c|}{$5-8$} & \multicolumn{4}{|c|}{$9-12$} & \multicolumn{2}{|c|}{$>12$} \\
\hline & $\%$ & \multicolumn{2}{|c|}{ IC 95\% } & $\%$ & \multicolumn{2}{|c|}{ IC 95\% } & $\%$ & \multicolumn{2}{|c|}{ IC 95\% } & $\%$ & \multicolumn{2}{|c|}{ IC 95\% } & $\%$ & \multicolumn{2}{|c|}{ IC 95\% } \\
\hline & 4 & 1,92 & 6,07 & 51 & 48,92 & 53,07 & 11 & 8,92 & 13,07 & 12,1 & 10,0 & 14,2 & 22 & 19,92 & 24,07 \\
\hline $\begin{array}{l}\text { Irregularmente } \\
\text { ativo }\end{array}$ & 2,7 & 0,62 & 4,77 & 39 & 36,92 & 41,07 & 10 & 7,92 & 12,07 & 26,0 & 23,9 & 28,1 & 22 & 19,92 & 24,07 \\
\hline Ativo & 3,3 & 1,22 & 5,37 & 34 & 31,92 & 36,07 & 18 & 15,92 & 20,07 & 25,3 & 23,2 & 27,4 & 20 & 17,92 & 22,07 \\
\hline Muito ativo & 1,3 & $-0,77$ & 3,37 & $65,6^{*}$ & 63,52 & 67,67 & 9 & 6,92 & 11,07 & 17,9 & 15,8 & 20,0 & $6,6^{*}$ & 4,52 & 8,67 \\
\hline
\end{tabular}

${ }^{*} \mathrm{p}<0,05$ diferença entre nível de atividade física (muito ativo) e anos de escolaridade (1-4 anos de estudo $>12$ anos de estudo).

Tabela 3: Porcentagem e intervalo de confiança do nível de atividade física da população de São Caetano do Sul de acordo com o bairro, São Caetano do Sul 2015.

\begin{tabular}{|c|c|c|c|c|c|c|c|c|c|c|c|c|}
\hline \multirow[b]{3}{*}{ Barcelona } & \multicolumn{3}{|c|}{ Sedentário } & \multicolumn{3}{|c|}{ Irreg. ativo } & \multicolumn{3}{|c|}{ Ativo } & \multicolumn{3}{|c|}{ Muito ativo } \\
\hline & \multirow{2}{*}{$\begin{array}{c}\% \\
19,6\end{array}$} & \multicolumn{2}{|c|}{ IC $95 \%$} & \multirow{2}{*}{$\begin{array}{c}\% \\
19,6\end{array}$} & \multicolumn{2}{|c|}{ IC 95\% } & \multirow{2}{*}{$\begin{array}{c}\% \\
41,1\end{array}$} & \multicolumn{2}{|c|}{ IC 95\% } & \multirow{2}{*}{$\begin{array}{c}\% \\
19,6\end{array}$} & \multicolumn{2}{|c|}{ IC 95\% } \\
\hline & & 17,5 & 21,7 & & 17,52 & 21,67 & & 39,0 & 43,17 & & 17,50 & 21,67 \\
\hline $\begin{array}{c}\text { Osvaldo } \\
\text { Cruz }\end{array}$ & 6,5 & 4,4 & 8,6 & 17,7 & 15,62 & 19,77 & 40,3 & 38,2 & 42,37 & 35,5 & 33,42 & 37,57 \\
\hline Mauá & 8,8 & 6,7 & 10,9 & 26,5 & 24,42 & 28,57 & 41,2 & 39,1 & 43,27 & 23,5 & 21,42 & 25,57 \\
\hline $\begin{array}{c}\text { Santo } \\
\text { Antônio }\end{array}$ & 21,7 & 19,6 & 23,8 & 28,3 & 26,22 & 30,27 & 41,3 & 39,1 & 43,27 & 8,7 & 6,62 & 10,77 \\
\hline Olímpico & 13,3 & 11,2 & 15,4 & 26,7 & 25,62 & 29,77 & 46,7 & 44,6 & 48,77 & 13,3 & 11,22 & 15,37 \\
\hline Santa Maria & 15,3 & 13,2 & 17,4 & 16,9 & 14,82 & 18,97 & 37,3 & 35,2 & 39,37 & 30,5 & 28,42 & 32,57 \\
\hline São Caetano & 17,6 & 15,5 & 19,7 & 17,6 & 15,52 & 19,67 & 47,1 & 45,0 & 49,17 & 17,6 & 15,52 & 19,67 \\
\hline Nova Gerty & 15,8 & 13,7 & 17,9 & 19,3 & 17,22 & 21,37 & 47,4 & 45,3 & 49,47 & 17,5 & 15,42 & 19,57 \\
\hline Santa Paula & 18,8 & 16,7 & 20,9 & 21,9 & 19,82 & 23,97 & 37,5 & 35,4 & 39,57 & 21,9 & 19,82 & 23,97 \\
\hline Boa Vista & 6,1 & 4,0 & 8,2 & 30,3 & 28,12 & 32,27 & 42,4 & 40,3 & 44,47 & 21,2 & 19,12 & 23,27 \\
\hline Cerâmica & 16,1 & 14,0 & 18,2 & 21,0 & 18,92 & 23,07 & 24,2 & 22,1 & 26,27 & 38,7 & 36,62 & 40,77 \\
\hline Centro & 8,7 & 6,6 & 10,8 & 30,4 & 28,32 & 32,47 & 47,8 & 45,7 & 49,87 & 13,0 & 10,92 & 15,07 \\
\hline Fundação & 12,1 & 10,0 & 14,2 & 33,3 & 31,22 & 35,37 & 45,5 & 43,4 & 47,57 & 9,1 & 7,02 & 11,17 \\
\hline Prosperidade & 6,2 & 4,1 & 8,3 & 37,5 & 35,42 & 39,57 & 25,0 & 22,9 & 27,07 & 31,2 & 29,12 & 33,27 \\
\hline São José & 23,4 & 21,3 & 25,5 & 10,6 & 7,92 & 12,07 & 42,6 & 40,5 & 44,67 & 23,4 & 21,32 & 25,47 \\
\hline
\end{tabular}




\section{Discussão}

Nesta pesquisa não foi possível observar diferenças estatisticamente significantes entre o nível de atividade física de acordo com os bairros de São Caetano do Sul. Entretanto, foi possível observar diferenças entre as classes socioeconômicas e entre a escolaridade e nível de atividade física.

Também cabe destacar que São Caetano do Sul ocupa a primeira posição entre os municípios com melhores Índices de Desenvolvimento Humano Municipal (IDHM), que o classifica com "muito alto desenvolvimento humano", apresentando valores muito diferentes dos demais em outros municípios em relação a ambiente e atividade física.

É importante salientar que não foi possível nesta pesquisa avaliar o ambiente que poderia influenciar no nível de atividade física, levando em consideração acessibilidade, segurança, áreas construídas, iluminação pública entre outros.

As evidências sobre estudos que relacionam ambiente construído e prática de atividades físicas no Brasil são escassas, principalmente em regióes com elevados índices de desenvolvimento humano. Entretanto, um estudo sobre ambiente construído e prática de atividade física em uma regiáo de São Paulo de baixo nível socioeconômico encontrou uma correlação com a quantidade de ambientes construídos e a prática regular de atividade física, principalmente no lazer ${ }^{13}$.

Em uma cidade do interior de São Paulo foi realizada uma pesquisa para encontrar a variável que mais tinha associação com a prática da atividade física no lazer e no transporte, os resultados mostraram que a quantidade de escolas, ciclovias e locais públicos não foram suficientes para aumentar a prática de atividade física nessa população ${ }^{14}$.

Em relaçáo ao sexo, estudo realizado no estado de São Paulo encontrou resultados semelhantes ao nível de atividade física entre homens e mulheres. O estudo apontou que a proporção de mulheres no grupo ativo foi de $48,6 \%$, já nos homens essa proporção foram menor apresentando apenas $42,5 \%$, esses dados corroboram nossos achados ${ }^{15}$.

No estado de João Pessoa na Paraíba que é classificada com "alto índice de desenvolvimento municipal", foi encontrado o inverso de nossos achados em relaçáo à escolaridade dos frequentadores de ambientes públicos. O maior nível de atividade física era praticado pelo grupo com maior quantidade de anos de estudo, sendo $64,4 \%$ com Ensino Superior e apenas 32,6\% com Ensino Fundamental e Médio ${ }^{16}$.

A prática de atividade física de acordo com o nível socioeconômico apresenta diferenças importantes visto que a promoção de saúde deveria atingir todos, independente de seu status econômico, entretanto esses resultados podem ser vistos como falha no sistema de promover saúde para os grupos mais desfavorecidos. Neste sentido a criação de políticas públicas deveriam abordar essas diferenças, conhecer os fatores e criar açôes especifica que proporcione de fato um ambiente favorável a pratica de atividades físicas para a manutenção da saúde da população.

\section{Conclusão}

Nesta pesquisa buscamos analisar a prevalência de atividade física nos 15 bairros da cidade de São Caetano do Sul, considerada a cidade com melhor IDH do Brasil. Com base nos dados analisados, concluímos que não apresentaram diferenças estatisticamente significantes entre o nível de atividade física de acordo com os bairros estudados, entretanto, foi possível observar diferenças entre as classes socioeconômicas e entre a escolaridade e nível de atividade física.

No entanto, algumas limitaçôes devem ser ressaltadas. Por se tratar de um grande inquérito de base populacional que investigou o grande $\mathrm{ABC}$, composto de sete cidades, para esta pesquisa foi selecionada apenas a cidade de São Caetano do Sul. A medida do nível de atividade física medida indiretamente possui viés que pode subestimar os valores reais.

Por último, destacamos que outros estudos de caráter exploratório devem ser realizados para que seja investigada a relação do ambiente construído na cidade com a prática de atividades físicas. Acreditamos ainda que esta pesquisa pode ser útil para os gestores municipais avaliarem a atual prática de atividade física da população e promover essas práticas nos bairros que apresentaram maior proporção de sedentários.

\section{Referências}

1. Mason C, Katzmarzyk PT, Craig CL, Gauvin L. Mortality and self-rated health in Canada. J Phys Act Health. 2007 Oct;4(4):423-33.

2. Lee IM, Shiroma EJ, Lobelo F, Puska P, Blair SN, Katzmarzyk PT, et al. Effect of physical inactivity on major non-communicable diseases worldwide: an analysis of burden of disease and life expectancy. Lancet. 2012 Jul 21;380(9838):219-29.

3. MATSUDO V.K.R, T. L.; MATSUDO, S. M. M.; GUEDES, J. S. Usando a Gestão Móbile de Modelo Ecológico para Promover Atividade Física. Diagnóstico \& Tratamento. 2006;11:184-9.

4. Matsudo V.K.R The "Agita Sao Paulo" experience in promoting physical activity. West Indian Med J. 2002 Mar 7-10;51 Suppl 1:48-50. 
5. REGO R.A. Fatores de risco para doenças crônicas não-transmissíveis: inquérito domiciliar no Município de São Paulo, SP (Brasil). Metodologia e resultados preliminares. Rev Saúde públ, S Paulo. 1990;24:277-85.

6. Florindo AA, Hallal PC, Moura EC, Malta DC. Practice of physical activities and associated factors in adults, Brazil, 2006. Rev Saude Publica. 2009 Nov;43 Suppl 2:65-73.

7. Del Duca GF, Nahas MV, Silva DA, Hallal PC, Malta DC, Peres MA. Physical activity indicators in adults from a state capital in the South of Brazil: a comparison between telephone and face-to-face surveys. Cad Saude Publica. 2013 Oct;29(10):2119-29.

8. Knuth AG, Bacchieri G, Victora CG, Hallal PC. Changes in physical activity among Brazilian adults over a 5-year period. J Epidemiol Community Health. 2010 Jul;64(7):591-5.

9. Matsudo VK, Matsudo SM, Araujo TL, Andrade DR, Oliveira LC, Hallal PC. Time trends in physical activity in the state of Sao Paulo, Brazil: 2002-2008. Med Sci Sports Exerc. 2010 Dec;42(12):2231-6.

10. WHO. Global Recommendations on Physical Activity for Health. 2011.
11. Barreto SMea. Analysis of the Global Strategy on Diet, Physical Activity and Health of the World Health Organization. Epidemiologia e Serviços de Saúde. 2005;14(1):41-68.

12. Matsudo S.M.M. International physical activity questionnaire (IPAQ): study of validity and reability in Brazil. Rev bras ativ fís saúde. 2001;6:5-18.

13. Florindo A.A. Built environment score related to leisure-time physical activity: application in a region of low socioeconomic status. Revista Brasileira de Cineantropometria e Desempenho Humano. 2013;15:243-55.

14. Nakamura P.M. Association between walking during leisure time and in the transportation with built environmnet in adults of Rio Claro-SP city. Revista Brasileira de Atividade Física \& Saúde. 2013;18(4):424-34.

15. Matsudo S. Physical activity level of São Paulo State population: an analysis based on gender, age, socio-economic status, demographics and knowledge. Rev Bras Ciên e Mov. 2002;10(4):41-50.

16. Junior JSMea. Physical Activity Level and Sociodemographic Profile of Frequenters of Public Places for Physical Activity Performance in The City of João Pessoa-PB. Revista Brasileira de Ciências da Saúde. 2013 2014;15(3):349-56.

\section{Como citar este artigo:}

Lima JS, Matsudo VK, Ribeiro MCS. Nível de atividade física de acordo com os bairros da cidade de São Caetano do Sul, SP. Rev. Aten. Saúde. 2016;14(47): 25-30. 